\title{
Mini-Mental State Examination is superior to plasma glucose concentrations in monitoring patients with suspected hypoglycaemic disorders during the 72-hour fast
}

\author{
Peter Wiesli, Beat Schwegler, Beat Schmid, Giatgen A Spinas and Christoph Schmid \\ Department of Internal Medicine, Division of Endocrinology and Diabetes, University Hospital, CH-8091 Zurich, Switzerland
}

(Correspondence should be addressed to Peter Wiesli; Email: peter.wiesli@dim.usz.ch)

\begin{abstract}
Objective: To determine whether systematic evaluation of cognitive function by the Mini-Mental State Examination (MMSE) allows the objective detection and documentation of cognitive deterioration in patients referred for evaluation of suspected hypoglycaemic disorders by the 72-h fast.

Design: Prospective case series.

Methods: In 50 patients referred for evaluation of suspected hypoglycaemic disorders, the MMSE score (maximum 30 points) was assessed at the start and at the end of the fast.

Results: The fast was terminated before $72 \mathrm{~h}$ in 14 patients because they developed neuroglycopenic symptoms due to hypoglycaemic disorders. Their MMSE score fell from a median of 29 points (range $20-30$ ) at the beginning to 17 points (range $0-24$ ) at the termination of the fast. The score dropped by $\geq 6$ points in all patients with hypoglycaemic disorders. Median (range) plasma glucose concentration at the end of the fast was $2.1(1.1-2.5) \mathrm{mmol} / \mathrm{l}$. Thirty-six individuals developed no neuroglycopenic symptoms throughout the 72-h fast, their MMSE score remained between 27 and 30 throughout the fast and their median plasma glucose concentration dropped to 2.9 (2-3.6) $\mathrm{mmol} / \mathrm{l}$. Conclusions: Systematic evaluation of cognitive function by the MMSE at the beginning and at the termination of the fast allows objective determination and documentation of the deterioration of the cognitive state in patients with hypoglycaemic disorders. A decline in the cognitive performance by $\geq 6$ points in the MMSE score rather than a distinct plasma glucose concentration should be used as the criterion to terminate the prolonged fast before $72 \mathrm{~h}$.
\end{abstract}

European Journal of Endocrinology 152 605-610

\section{Introduction}

The classic time point for termination of the 72-h fast in patients evaluated for hypoglycaemic disorders is the occurrence of hypoglycaemic symptoms in association with a low plasma glucose level (1). However, neither the symptoms nor the plasma glucose cut-off value have been exactly defined in prospective studies. We have demonstrated that terminating the fast at plasma glucose levels between 2.1 and $2.5 \mathrm{mmol} / \mathrm{l}$ in the absence of neuroglycopenic symptoms does not allow an insulin-secreting tumour to be confirmed or excluded because glucose concentrations and insulin levels in healthy individuals and patients with insulinsecreting tumours may overlap, even at plasma glucose concentrations lower than $2.5 \mathrm{mmol} / \mathrm{l}$ (2). Therefore, the occurrence of neuroglycopenic symptoms is the prerequisite criterion to end the fast.

Severe hypoglycaemia results in impaired cognitive function $(3,4)$. Cognitive functions that deteriorate in hypoglycaemia include simple and choice reaction times, arithmetric calculation, verbal fluency, colour identification, attention, concentration, short-term memory, information processing and mood state $(5,6)$. Comprehensive assessment of these cognitive domains requires complex neuropsychological test batteries. However, it would not be practical to serially perform complex neuropsychological tests during the 72-h fast in patients evaluated for hypoglycaemic disorders. The Mini-Mental State Examination (MMSE) is a standardised test which was proposed as a simple and quickly administered method for grading cognitive function (7). The MMSE is the most widely used cognitive screening instrument for the detection of cognitive dysfunction due to dementia in geriatric and psychiatric patients (8). The MMSE evaluates orientation, memory, attention and language functions.

The purpose of this study was to assess whether systematic evaluation of the cognitive function by the MMSE at the beginning and termination of the fast may be used to detect cognitive deterioration objectively in patients evaluated for hypoglycaemic disorders. 


\section{Subjects and methods}

\section{Subjects}

Fifty patients (35 women and 15 men) who underwent a prolonged fast (between May 2001 and October 2004) for the evaluation of a suspected hypoglycaemic disorder were included. Median age was 48 years (range 16-84 years) and body mass index $22.4 \mathrm{~kg} / \mathrm{m}^{2}$ (range $18-35.6 \mathrm{~kg} / \mathrm{m}^{2}$ ). Screening for sulphonylureas in the blood at the termination of the fast was negative in all patients. The Ethics Committee of the University Hospital of Zurich decided that this study did not need approval since the protocol included no more than standard care and involved no additional hazard to patients. Oral informed consent was obtained from all patients after the procedure was fully explained.

\section{The 72-h fast}

Patients were hospitalised for the supervised 72-h fast, which was performed following a standardised protocol as previously described (2). Non-essential medication was discontinued. Patients were allowed to drink only calorie-free and caffeine-free beverages during the 72-h fast. Blood samples were collected every $4 \mathrm{~h}$ for determination of plasma glucose and insulin. The criterion to end the fast before $72 \mathrm{~h}$ was the occurrence of clearly defined neuroglycopenic signs and symptoms which were repetitively assessed by interviewing and examining the patient. If the patient was considered to exhibit neuroglycopenic symptoms, the MMSE was performed. Plasma glucose and serum insulin concentrations were determined at the termination of the fast. In addition, plasma samples were screened for first- and second-generation sulphonylureas to rule out factitious hypoglycaemia. The biochemical results (insulin, C-peptide, proinsulin and $\beta$-hydroxybutyrate concentrations) at the termination of the fast of 11 patients with hypoglycaemic disorders and 18 healthy individuals have been shown previously (9).

\section{Cognitive evaluation}

The MMSE was performed at normal glucose levels the day before the fast or within the first hour of the fast, as well as at the end of the fast. The MMSE questionnaire assesses orientation in time and space, immediate and delayed verbal memory, attention and calculation, constructional capacity and language (7). The 30 test items were each scored by one point if successfully completed; incorrectly completed items received a zero score. Incapacitated patients unable to complete all 30 items received one point for each missed item. The performance score in the MMSE ranges from 0 to 30 points, with lower scores indicating greater impairment of cognitive function.

\section{Laboratory investigations}

Venous blood samples were drawn into sodium fluoridecontaining tubes for the determination of plasma glucose. Plasma glucose was measured by the glucose oxidase technique (Beckman Analyzer; Beckman, Fullerton, CA, USA). Samples for the determination of glucose and insulin were immediately centrifuged. Immunoreactive insulin was measured by solid-phase RIA (intra-assay coefficient of variation (CV), 5\%; inter-assay CV 8.5\%) (CIS Bio international, Oris Industries, Gif-Sur-Yvette, France) with a cross-reactivity of $14 \%$ for proinsulin and $0.1 \%$ for C-peptide as previously described (10).

\section{Results}

\section{Subjects}

In 14 patients (out of the 50 subjects included) the fast was prematurely terminated after a median of $23 \mathrm{~h}$ (range 8-60 h) because of neuroglycopenic symptoms at venous plasma glucose levels of 2.1 (1.3-2.5) $\mathrm{mmol} / \mathrm{l}$. An insulin concentration of $165 \mathrm{pmol} / \mathrm{l}$ (range $74-340 \mathrm{pmol} / \mathrm{l}$ ) and C-peptide concentration of $905 \mathrm{pmol} / \mathrm{l}$ (range $310-2050 \mathrm{pmol} / \mathrm{l}$ ) at the termination of the fast indicated endogenous hyperinsulinaemic hypoglycaemia. An insulin-secreting tumour was localised by selective arterial calcium stimulation and hepatic venous sampling techniques in 12 patients (11). These patients were referred to surgery. Insulinsecreting tumours were successfully removed and the diagnosis of an insulinoma was confirmed histologically. One patient with hyperinsulinaemic hypoglycaemia was found to have nesidioblastosis. This patient underwent distal pancreatic resection. A postoperatively performed $72-\mathrm{h}$ fast confirmed the absence of neuroglycopenic symptoms throughout $72 \mathrm{~h}$ of fasting (the results of this second fast are not included in this study). On follow-up, no hypoglycaemic episodes reoccurred in these 13 patients. In one patient, hypoglycaemia was caused by an islet cell carcinoma with liver metastases. Diagnosis was confirmed in this patient by liver biopsy.

Thirty-six individuals exhibited no symptoms or signs of neuroglycopenia throughout $72 \mathrm{~h}$ of fasting. Median plasma glucose concentration in these individuals dropped to $2.9(2-3.6) \mathrm{mmol} / \mathrm{l}$. Insulin levels (46 pmol/l, range $15-109 \mathrm{pmol} / \mathrm{l})$ at the end of the fast were in an appropriate range in these 36 patients. An insulin-secreting tumour was excluded in these individuals because of the absence of neuroglycopenic symptoms during the fast as well as appropriately decreased insulin (and C-peptide) concentrations at the end of the fast. Moreover, an oral glucose challenge test was performed in most of these patients without evidence for reactive hypoglycaemia. During follow-up, no hospital admissions or emergency room visits occurred due to hypoglycaemia in these 36 patients. 


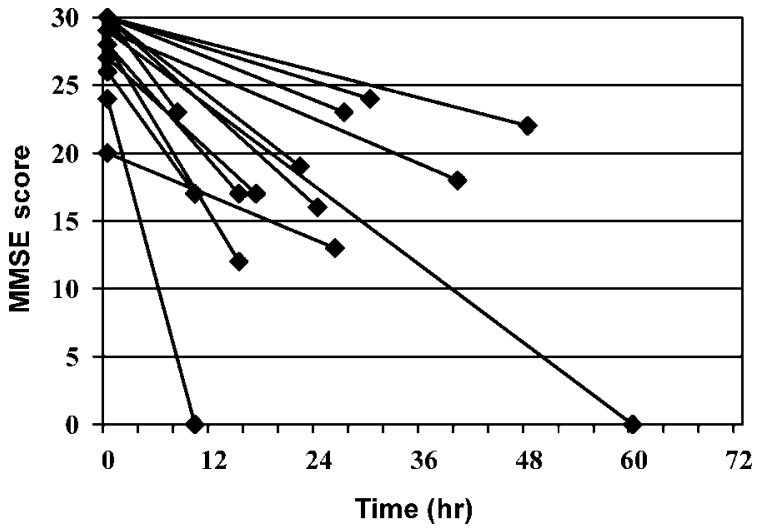

Figure 1 The scores of the MMSE at the beginning and termination of the fast in 14 patients with hypoglycaemic disorders, connected by a straight line for each individual patient (not reflecting the true time course).

\section{Cognitive evaluation in 14 patients with neuroglycopenic symptoms during the fast}

In 14 patients the fast was terminated because of neuroglycopenic symptoms. The MMSE score fell from a median of 29 points (range $20-30$ points) at the beginning to 17 points (range $0-24$ points) at the termination of the fast (Fig. 1). Median plasma glucose levels at the initial assessment of the cognitive state was $4.5(3.9-9.6) \mathrm{mmol} / \mathrm{l}$ and $2.1(1.1-2.5) \mathrm{mmol} / \mathrm{l}$ at the termination of the fast (data at the end of the fast are shown in Fig. 2). The detailed results of the MMSE in the patients with neuroglycopenic symptoms during the fast are given in Table 1. The total MMSE score reliably dropped (by $\geq 6$ points) in all patients during neuroglycopenia. Calculation and attention was impaired in all patients, whereas the cognitive evaluation for orientation in time and space, immediate recall, delayed verbal memory, and language disclosed variable results.

\section{Cognitive evaluation in 36 patients without neuroglycopenic symptoms throughout the 72-h fast}

Thirty-six patients did not exhibit neuroglycopenic symptoms during the 72-h fast. The MMSE score remained unchanged in the range of 27-30 points throughout the fast. Mean plasma glucose levels at the initial assessment of the cognitive state were 4.7 $(3.9-7.1) \mathrm{mmol} / \mathrm{l}$ and were $2.9(2-3.6) \mathrm{mmol} / \mathrm{l}$ at the termination of the fast (Fig. 2). One patient had a drop in MMSE score of two points, in five patients the drop in the MMSE score was one point. In thirty patients, performance in the MMSE during the fast was unchanged or even improved by one or two points.

\section{Glucose concentration, insulin concentration, insulin to glucose ratio and the drop in the MMSE score in patients with and without neuroglycopenic symptoms}

In Fig. 2, the drop in the MMSE score, plasma glucose concentrations, serum insulin concentrations and insulin to glucose ratios in patients with (A in Fig. 2) and without (B in Fig. 2) neuroglycopenic symptoms during the fast are shown. The MMSE score separates the two groups of patients by a drop in the score of $\geq 6$ points; in contrast, glucose concentrations, insulin concentrations, and insulin to glucose ratios at the end of the fast overlap in patients with and without hypoglycaemic disorders. The sensitivities and specificities for the decrease in the MMSE score and different plasma glucose concentrations to
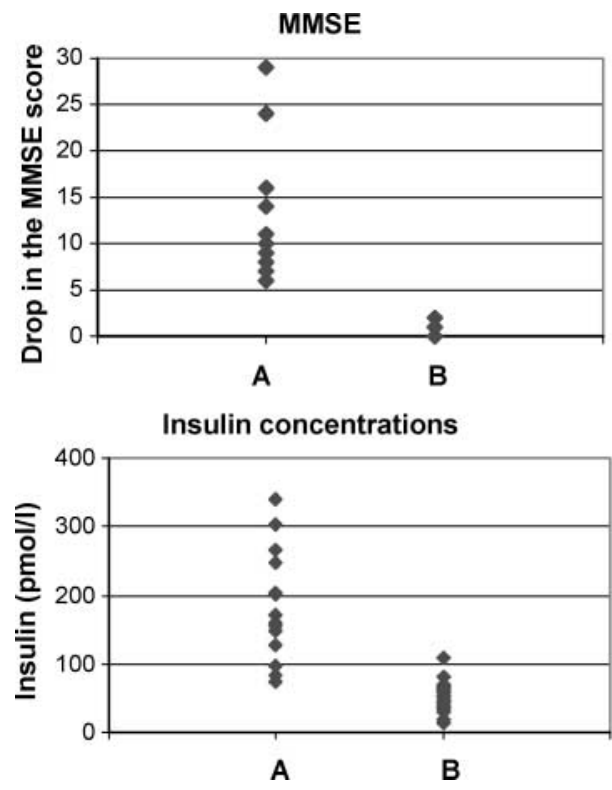
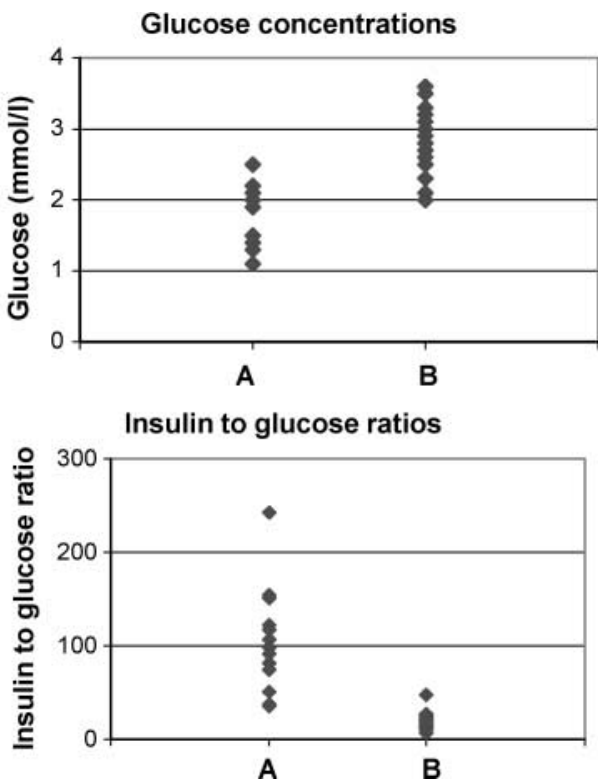

Figure 2 The drop in the MMSE score, plasma glucose, serum insulin concentrations and insulin (pmol/l) to glucose $(\mathrm{mmol} / \mathrm{l})$ ratios at the termination of the prolonged fast in 14 patients with hypoglycaemic disorders (A) and 36 individuals without hyperinsulinaemic hypoglycaemia (B). 
Table 1 Cognitive function assessed by MMSE in 14 patients who exhibited neuroglycopenic symptoms during the fast. Median (range) blood glucose concentration was 4.5 (3.9-9.6) $\mathrm{mmol} / \mathrm{l}$ at the beginning and $2.1(1.1-2.5) \mathrm{mmol} / \mathrm{l}$ at the termination of the fast.

\begin{tabular}{lccc}
\hline MMSE & $\begin{array}{c}\text { Maximum } \\
\text { score }\end{array}$ & $\begin{array}{c}\text { Median score at } \\
\text { the beginning } \\
\text { (range) }\end{array}$ & $\begin{array}{c}\text { Median score at } \\
\text { the termination } \\
\text { (range) }\end{array}$ \\
\hline $\begin{array}{c}\text { Orientation } \\
\text { in time }\end{array}$ & 5 & $5(2-5)$ & $2.5(0-5)$ \\
$\begin{array}{c}\text { Orientation } \\
\text { in space }\end{array}$ & 5 & $5(4-5)$ & $4(0-5)$ \\
$\begin{array}{l}\text { Immediate } \\
\text { recall }\end{array}$ & 3 & 3 & $3(0-3)$ \\
$\begin{array}{c}\text { Delayed verbal } \\
\text { memory }\end{array}$ & 3 & $2.5(0-3)$ & $0(0-3)$ \\
$\begin{array}{c}\text { Attention and } \\
\text { calculation }\end{array}$ & 5 & $5(1-5)$ & $1.5(0-3)$ \\
$\begin{array}{l}\text { Language } \\
\text { Total MMSE }\end{array}$ & 9 & $9(7-9)$ & $6.5(0-9)$ \\
\hline
\end{tabular}

diagnose a hypoglycaemic disorder are shown in Table 2.

\section{Discussion}

Our data demonstrate that MMSE scores reliably drop (by $\geq 6$ points) in patients with hypoglycaemic disorders during the 72-h fast, whereas plasma glucose concentrations at the termination of the fast overlap with those of healthy individuals. A decline in the MMSE score by $\geq 6$ points during the prolonged fast had a sensitivity of $100 \%$ and specificity of $100 \%$ for diagnosis of a hypoglycaemic disorder; in contrast, sensitivities and specificities of different plasma glucose concentrations between 2 and $2.5 \mathrm{mmol} / \mathrm{l}$ were lower (Table 2). Thus, a decline in the MMSE score rather than a distinct plasma glucose concentration should be used as the criterion to end the prolonged fast. The MMSE can be performed quickly, requiring only a few minutes to complete. The test can be repeated as serial measurement to document the crucial aspect of mental functioning during the fast. A decline in the score clearly indicates deterioration of cognitive function. If anything, performance in the MMSE would be expected to improve due to a practice effect when the test is serially conducted. The potential to compare the result of the cognitive evaluation at the termination

Table 2 Sensitivities and specificities of the change in the MMSE score and different plasma glucose concentrations at the termination of a fast to diagnose a hypoglycaemic disorder.

\begin{tabular}{llcc}
\hline Criterion & \multicolumn{1}{c}{ Cut-off } & Sensitivity & Specificity \\
\hline Drop in MMSE score & $\geq 6$ points & $100 \%$ & $100 \%$ \\
Plasma glucose & $<2 \mathrm{mmol} / \mathrm{l}$ & $36 \%$ & $100 \%$ \\
Plasma glucose & $\leq 2 \mathrm{mmol} / \mathrm{l}$ & $50 \%$ & $91 \%$ \\
Plasma glucose & $\leq 2.2 \mathrm{mmol} / \mathrm{l}$ & $93 \%$ & $88 \%$ \\
Plasma glucose & $\leq 2.5 \mathrm{mmol} / \mathrm{l}$ & $100 \%$ & $68 \%$ \\
\hline
\end{tabular}

of the fast with the initial test at normal glucose concentrations could also be helpful in older patients with cognitive impairment at the beginning of the test. However, we had the opportunity to test only one individual with an insulinoma and impaired cognitive functioning due to dementia. In this patient, baseline MMSE was performed twice; MMSE scores were 20 and 19 points respectively, and dropped to 13 points at a venous plasma glucose concentration of $2.5 \mathrm{mmol} / \mathrm{l}$.

The fast lasted $72 \mathrm{~h}$ for the 36 individuals without symptoms or signs of neuroglycopenia during the test. Although plasma glucose concentrations dropped to levels as low as $2 \mathrm{mmol} / \mathrm{l}$ in several healthy individuals, the MMSE score remained in a range between 27 and 30 points throughout the fast. The overlapping glucose levels of healthy individuals and patients with hypoglycaemic disorders in our study are all the more remarkable since venous plasma glucose was assessed: a potential arterio-venous glucose gradient would be expected to be higher in the insulinoma patients than in the healthy individuals with suppressed insulin concentrations. The explanation as to why healthy individuals do not develop cognitive impairment at plasma glucose concentrations at which patients with hyperinsulinaemic hypoglycaemic disorders may exhibit a marked drop in the MMSE score cannot be derived from our study. To our knowledge, there are no convincing data linking specific spontaneous venous plasma glucose values to cognitive impairment in patients with hypoglycaemic disorders. Studies using the setting of a stepped hypoglycaemic-clamp (peripheral insulin administration) and monitoring arterialised venous blood samples have indicated that performance of cognitive tests deteriorated significantly in healthy volunteers when insulin was infused to reach plasma glucose levels of $2.3 \mathrm{mmol} / \mathrm{l}$; the decline in cognitive function was estimated to begin at $2.7 \mathrm{mmol} / \mathrm{l}$ (3). Using well-defined experimental protocols in healthy volunteers (and in patients with type 1 diabetes), estimated glycaemic thresholds resulting in impaired cognitive function were lower following antecedent hypoglycaemia, suggesting adaptation as also described for neuroendocrine counterregulation (12). It is possible that type 1 diabetics and longer-term fasting healthy individuals with recurrent hypoglycaemia share at least in part cerebral adaptation by up-regulating GLUT-1 and GLUT-3 in the brain, a process which requires time. Depending on the time frame within which blood glucose decreases, an additional type of adaptation may come into play for healthy individuals (but not for insulinoma patients following an excessive burst of insulin secretion); i.e. if their glucose level is slowly decreasing, the fuel mixture will change over time to provide additional appropriate energy sources for maintenance of brain function. This implies that the term 'glycaemic threshold' may not be applicable under these conditions; it is possible that the 'glycaemic thresholds' determined in laboratories overestimate the 
blood glucose levels at which brain function is significantly impaired, and a healthy individual may well tolerate lower glucose values in the situation of a longer-term fast. In contrast to healthy individuals with suppressed insulin concentrations during the fast, ketone bodies such as $\beta$-hydroxybutyrate are less available in insulinoma patients since insulin blocks lipolysis and ketogenesis in addition to hepatic glucose production (13).

In a previous study including six patients with insulinomas before surgery, cognitive function was systematically assessed during a stepped hypoglycaemic clamp (14). In this artificial setting, no significant change in cognitive function was observed until the lowest plasma glucose concentration of $2.2 \mathrm{mmol} / \mathrm{l}$. In our study, we demonstrate a striking deterioration of cognitive function in 14 patients with hypoglycaemic disorders (presumably due to excessive release of insulin into the portal vein). Apart from the fact that simply fasting allowed a further drop in plasma glucose than tested in that clamp study, a further explanation would be that the onset and duration of experimentally induced hypoglycaemia may be different from that of spontaneously occurring hypoglycaemia during the fast. Plasma glucose levels were maintained at the lowest level of $2.2 \mathrm{mmol} / \mathrm{l}$ for $45 \mathrm{~min}$ only in the study of Mitrakou et al. (14). In our study, all patients developed hypoglycaemia in an unpredictable manner after several hours, as pointed out by Berman et al. (15). This highlights the importance of carefully supervising the patients for neuroglycopenia during the fast and to end the fast not solely on the basis of low plasma glucose concentrations. As mentioned above, additional factors, apart from glucose deprivation, seem to be responsible for the development of the socalled 'neuroglycopenic' symptoms.

As illustrated in Fig. 2, patients with hypoglycaemic disorders and healthy individuals cannot be distinguished by venous plasma glucose concentrations, serum insulin concentrations or insulin to glucose ratios at the termination of the fast. Across the parameters monitored during the fast, the occurrence of neuroglycopenia during the fast was the best (and, moreover, the most readily available) criterion for discriminating patients with hypoglycaemic disorders from healthy individuals. A decline in the MMSE score by $\geq 6$ points had a $100 \%$ sensitivity and $100 \%$ specificity to detect patients with hypoglycaemic disorders. However, clinical decision making should not be based on one single criterion. Multiple clinical and biochemical criteria should be considered for diagnosis or exclusion of a hypoglycaemic disorder.

\section{Conclusions}

Systematic evaluation of cognitive function by the MMSE at the beginning and at the end of the fast allows the documentation of the cognitive state which deteriorates in severe hypoglycaemia. MMSE scores reliably drop when significant hypoglycaemia occurs during the fast. A decline in the MMSE score (by $\geq 6$ points) rather than a distinct plasma glucose concentration should be used as criterion to end the 72-h fast.

\section{Acknowledgements}

We thank Heidi Seiler for excellent technical assistance.

\section{References}

1 Service FJ. Hypoglycemic disorders. New England Journal of Medicine $19953321144-1152$.

2 Wiesli P, Brandle M, Schwegler B, Lehmann R, Spinas GA \& Schmid C. A plasma glucose concentration below $2.5 \mathrm{mmol} \mathrm{L}^{-1}$ is not an appropriate criterion to end the 72-h fast. Journal of Internal Medicine $2002252504-509$.

3 Mitrakou A, Ryan C, Veneman T, Mokan M, Jenssen T, Kiss I, Durrant J, Cryer P \& Gerich J. Hierarchy of glycemic thresholds for counterregulatory hormone secretion, symptoms, and cerebral dysfunction. American Journal of Physiology $19912 \mathbf{2 6 0}$ E67-E74.

4 Blackman JD, Towle VL, Sturis J, Lewis GF, Spire JP \& Polonsky KS. Hypoglycemic thresholds for cognitive dysfunction in IDDM. Diabetes $199241392-399$.

5 Draelos MT, Jacobson AM, Weinger K, Widom B, Ryan CM, Finkelstein DM \& Simonson DC. Cognitive function in patients with insulin-dependent diabetes mellitus during hyperglycemia and hypoglycemia. American Journal of Medicine $1995 \mathbf{9 8}$ 135-144.

6 Strachan MW, Deary IJ, Ewing FM \& Frier BM. Recovery of cognitive function and mood after severe hypoglycemia in adults with insulin-treated diabetes. Diabetes Care 200023 305-312.

7 Folstein MF, Folstein SE \& Mchugh PR. 'Mini-mental state'. A practical method for grading the cognitive state of patients for the clinician. Journal of Psychiatric Research 197512 189-198.

8 Tombaugh TN \& Mcintyre NJ. The mini-mental state examination: a comprehensive review. Journal of the American Geriatric Society $1992 \mathbf{4 0} 922-935$.

9 Wiesli P, Brandle M, Zapf J, Seiler H, Zwimpfer C, Spinas GA \& Schmid C. Assessment of hyperinsulinaemia at the termination of the prolonged fast. Clinica et Chimica Acta $2004 \mathbf{3 4 2}$ 227-231.

10 Wiesli P, Brandle M, Pfammatter T, Zapf J, Spinas GA \& Schmid C. Insulin determination by specific and unspecific immunoassays in patients with insulinoma evaluated by the arterial stimulation and venous sampling test. European Journal of Endocrinology 2004151 123-126.

11 Wiesli P, Brandle M, Schmid C, Krahenbuhl L, Furrer J, Keller U, Spinas GA \& Pfammatter T. Selective arterial calcium stimulation and hepatic venous sampling in the evaluation of hyperinsulinemic hypoglycemia: potential and limitations. Journal of Vascular and Interventional Radiology 200415 $1251-1256$.

12 Schultes B, Kern W, Oltmanns K, Peters A, Gais S, Fehm HL \& Born J. Differential adaptation of neurocognitive brain functions to recurrent hypoglycemia in healthy men. Psychoneuroendocrinology 200530 149-161.

13 Rizza RA, Haymond MW, Verdonk CA, Mandarino LJ, Miles JM, Service FJ \& Gerich JE. Pathogenesis of hypoglycemia in 
insulinoma patients: suppression of hepatic glucose production by insulin. Diabetes 198130 377-381.

14 Mitrakou A, Fanelli C, Veneman T, Perriello G, Calderone S, Platanisiotis D, Rambotti A, Raptis S, Brunetti P \& Cryer P. Reversibility of unawareness of hypoglycemia in patients with insulinomas. New England Journal of Medicine 1993329 834-839.

15 Berman N, Genter P, Chou HF, Cortez C, Bowsher R \& Ipp E. Erratic oscillatory characteristics of plasma insulin concentrations in patients with insulinoma: mechanism for unpredictable hypoglycemia. Journal of Clinical Endocrinology and Metabolism 199782 2899-2903.

Received 17 November 2004

Accepted 19 January 2005 